\title{
Fatigue crack growth law identification by Digital Image Correlation and electrical potential method for ductile cast iron
}

\author{
J. Hosdez, J-F. Witz, C. Martel, N. Limodin, D. NajJar, \\ E. Charkaluk, P. Osmond and F. SzmytKa* ${ }^{+}$ \\ Centrale Lille, Cite Scientifique, 59651 Villeneuve d'Ascq, France. \\ Laboratoire de Mecanique de Lille (LML), FRE CNRS 3723, Cite Scientifique, 59651 Villeneuve d'Ascq, France. \\ PSA Peugeot Citroen, Route de Gisy, 78140 Velizy-Villacoublay Cedex, France.
}

\begin{abstract}
In this paper, a comparison between two methods used to identify fatigue crack propagation law is conducted: Digital Image Correlation (DIC) and Direct Current Potential Drop (DCPD). For this purpose, fatigue tests were conducted at R-ratio of 0.1 on a ductile cast iron commonly used for exhaust manifolds manufacturing. Results show a good agreement between the methods illustrating the accuracy of each technique for the analysis of fatigue crack growth. Moreover, an interest of DIC is also to allow studying the plasticity that occurs at the crack tip during the fatigue test.
\end{abstract}

Keywords: Digital Image Correlation, Direct Current Potential Drop, Fatigue, Stress Intensity Factor, Paris law, Plasticity

\section{INTRODUCTION}

$\coprod^{N}$ $\mathrm{N}$ the automotive industry, significant effort is devoted to satisfying the new legal environmental rules and to reduce weight. These constraints lead to a more demanding use of material, especially in case of engine components submitted to important mechanical loadings (thermo-mechanical fatigue, creep, fatigue induced by vibrations). In the area of fatigue design, mechanical reliability is one of the most important challenges, in spite of over 150 years of research into fatigue life prediction [1]. Designing against fatigue failure conducted to engineering rules such as the S-N curve or simplified equations as Basquin [2] for HCF (High Cycle Fatigue) and Manson-Coffin [3] [4] for LCF (Low Cycle Fatigue). Nevertheless, the fatigue lifetime of an engine part is related to the crack initiation, its growth at microscale (short cracks) and then at structure scale (long cracks) until failure.

With the emergence of fracture mechanics, a more ambitious task has been undertaken the prediction of the propagation behaviour of fatigue cracks. This study is based on fracture mechanics concepts, developed from 1920. Griffith [5] showed that the fracture of an elasticbrittle medium can be characterized by a variable, called later energy release rate. In the forties, Westergaard [6] and Muskhelishvili [7] gave analytical formulas of mechanical fields close to a

\footnotetext{
${ }^{*}$ Correspondence to: J. Hosdez, Centrale Lille, Cite Scientifique, 59651 Villeneuve d'Ascq, France.

${ }^{\dagger}$ E-mail: jerome.hosdez@ec-lille.fr
} 
crack in a linear elastic medium. In 1956, Irwin [8] and Williams [9], from singularities fields studies, defined the Stress Intensity Factor (SIF) notion to quantify singularity at the crack tip for the three cracking modes $\left(K_{I}, K_{I I}, K_{I I I}\right)$. Under certain conditions, Williams characterized the displacement field near the crack front with expansion series distinguishing the contributions of each cracking mode. In the linear elastic fracture mechanics framework, Paris and Erdogan [10] studied the propagation of long fatigue cracks and showed that the propagation velocity is clearly not constant in time and depends on the SIF range $\Delta K$. During the stable crack propagation, also called the Paris regime, the crack growth rate $d a / d N$ is a power function of the SIF range leading to the well-known Paris equation:

$$
d a / d N=C(\Delta K)^{m}
$$

where $C$ and $m$ are experimentally obtained constants. Thus, the law identification requires to assess the crack length and SIFs values.

The first step is to experimentally determine the crack geometry with an accurate measurement of the crack length (or the crack tip position). Several methods can be used such as post mortem fatigue striations observation, but some materials such as the ductile cast irons studied here do not allow them to be directly observed [11]. Fatigue crack front marking can be expected with overloads, load ratio modifications or with heat tinting [12], taking the risk to impact the crack velocity. Among all the methods, the electrical [13] and optical monitoring are particularly interesting because a continuous follow-up is possible and a measurements automation can be considered. On the one hand, it eliminates uncertainties due to perception/detection of the crack tip location by the user and on the other hand, it avoids holding times. Indeed, some transitory effects or a residual creep contribution, especially at high temperature, could affect the crack behavior and the crack growth law identification. Moreover, these approaches enable to increase the acquisition frequency. The electrical potential difference such as Direct Current Potential Drop (DCPD) method, consists in applying an electrical current through a specimen and to measure the potential difference between the crack lips. The variation in potential drop can be related to the crack length evolution, providing an appropriate calibration [12] [13].

The second step is the SIFs measurement, for which analytical formulas exist in Murakami's [14] and Tada, Paris and Irwin's handbooks [15]. SIFs expressions are given as function of the applied load and specimen dimensions. However, these expressions are valid only for the considered specimen. A substitute approach commonly used is the Finite Elements Method (FEM), where SIFs are calculated using J-integral [16] or interaction integrals [17]. Finally, Digital Image Correlation (DIC) has also been used for a wide range of applications and material systems as in [18] with the study of fatigue surface damage or in [19] to appreciate crack closure effects. With this technique, experimental displacement fields, at the vicinity of the crack tip, can be fitted with a theoretical model such as Williams series allowing SIFs extraction with least squares method [20] [21]. This approach was retained to control the stress intensity factor during a fatigue crack propagation in 2D [22] and it has also been used in 3D for through cracks with nearly straight front [23] and part-through cracks with curved fronts [24]. Recently, a unified framework coupling the latter method with FEM calculations was proposed, where Paris law is identified and then validated by simulating the experiment [25]. An alternative strategy is the integrated digital image correlation (iDIC), where displacement measurement and behavior identification are identified at once [26]. 
The objective of the present study is to compare the Paris law identifications using two alternative methods namely the DCPD method coupled with Finite Elements calculations and a projective method based on DIC measurements projected on Williams series. Moreover, an iDIC procedure will be compared to these two techniques. These approaches are applied to a constant amplitude loading test. The studied material is a cast iron commonly used for exhaust manifolds manufacturing, parts often subjected to fatigue cracks [27] [28].

\section{IDENTIFICATION PROCESS}

This study was undertaken for a comparison between different experimental resources to identify the crack growth law. The first one is the Direct Current Potential Drop (DCPD) method with a numerical calibration and the second one is the Digital Image Correlation coupled with a projection on a specific basis. Limitations of each method will be shown after presenting the main guidelines for their use. Firstly, an overview of the experimental facilities will be made, after which the methods of identification will be presented.

\section{Direct Current Potential Drop method}

The Direct Current Potential Drop (DCPD) method allows the measurement of crack length during a fatigue crack growth test for both long [29] or short cracks [12] with a resolution on crack length increment detection close to $50 \mu \mathrm{m}$ [30]. The principle is to apply a constant electrical current across the specimen and to measure the potential difference through the crack. When the crack extends, the electrical resistance will increase and the measured potential as well. This method is especially attractive for its stability, repeatability and when no optical access is possible allowing its use in a closed environment [13]. But, as it is an indirect method, it needs a calibration to establish the relation between the electrical potential and the crack size. The potential will depend on the crack length and depth but also on the specimen geometry, the potential probes and current leads locations, electrical resistivity of the material and temperature.

There are several calibration methods such as optical methods, fracture surface observations after load path modification or with heat tinting [12], Finite Element Method (FEM) [31] and analytical law with the Johnson's formula amply used and improved over the years [32] [13]. The DCPD method accuracy will mostly depend on the calibration curve [12] and its sensitivity will increase when the measuring probes are closer to the crack [33]. Besides, to obtain accurate results, it is necessary to take some precautions about the electrical insulation or the stability and sensitivity of the measurement systems. It is required to wait for a steady-state before commencing the test to account for heat generation or transition effects. It can be observed that a five hour wait is enough to reach a permanent state.

\section{Digital image correlation}

Digital Image Correlation (DIC) is a technique that allows displacements to be measured on surfaces under different loading conditions [34]. If grey levels conservation is supposed, the difference between the pictures taken at different instants depends only on displacements field:

$$
f(\bar{x})=g(\bar{x}+\bar{u}(\bar{x}))
$$

with $f(\bar{x})$ and $g(\bar{x})$, respectively the reference and deformed states seen as scalars (grey levels) and $\bar{u}$ is the displacement vector for each position $\bar{x}$. Finding the best $\bar{u}$ field is achieved by 
minimizing the optical flow equation (Eq. (3)) over the Region Of Interest (ROI) $\Omega$ :

$$
\eta^{2}=\int_{\Omega}[g(\bar{x}+\bar{u}(\bar{x}))-f(\bar{x})]^{2} d x
$$

DIC is done with YaDICs software, developed in Laboratoire de Mecanique de Lille [35]. To identify displacements, several parameters have to be defined: a metric, a sampling, an interpolator, a transformation, an optimizer and finally a regularization method (Fig. 1).

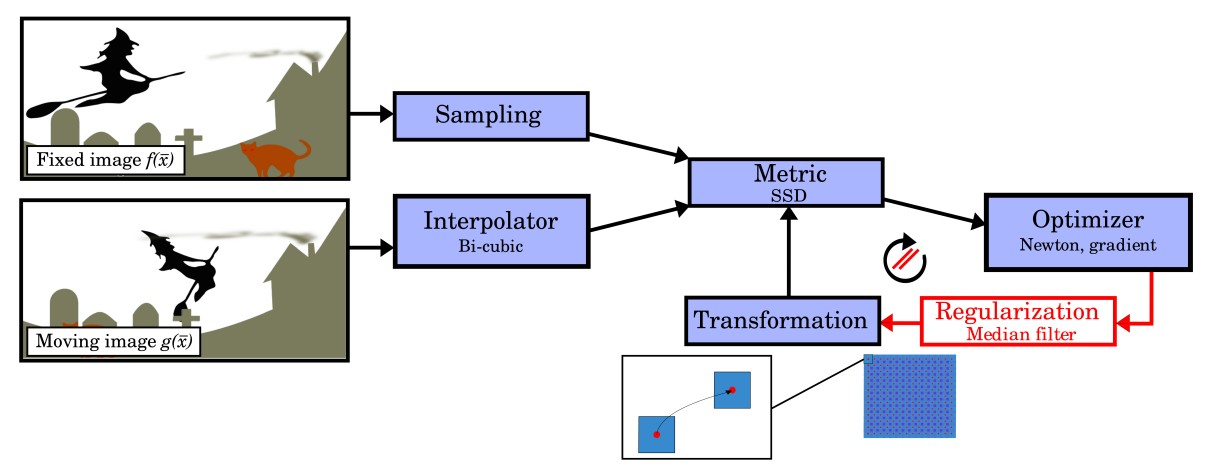

Figure 1: Different steps of the DIC process with YaDICs software [35].

In this study, rigid local transformation is selected in order to compute the chosen Sum Squared Difference (SSD) metric. This latter is evaluated on the whole image and the gradient of the metric is computed regarding the transform parameters (Eq. (4)), and the optimization is performed with an iterative numerical algorithm (Eq. (5)).

$$
\begin{gathered}
\frac{\partial S S D}{\partial \bar{u}}=\frac{2}{\Omega} \sum_{\bar{x} \in \Omega}(f(\bar{x})-g(\bar{x}+\bar{u}(\bar{x}))) \frac{\partial \bar{g}(\bar{x}+\bar{u}(\bar{x}))}{\partial \bar{u}} \\
\bar{u}_{k+1}=\bar{u}_{k}+\alpha_{k} \bar{d}_{k}
\end{gathered}
$$
by:

with $\alpha_{k}$, the gradient step which can be constant or updated at every iteration and $\bar{d}_{k}$ is defined

$$
\begin{gathered}
\bar{d}_{k}=-\gamma_{k} \frac{\partial S S D(\bar{u}, f, g)}{\partial \bar{u}} \\
\gamma_{k}=\left[\frac{\partial S S D}{\partial \bar{u}} \cdot \frac{\partial S S D^{t}}{\partial \bar{u}}\right]^{-1}
\end{gathered}
$$

Finally, the evaluation of the moving image grey levels at the coordinates given by the transformation is performed using a bi-cubic interpolation.

The correlation procedure is based on a multiscale resolution strategy, i.e. a pyramid scheme. The "scale 0 " corresponds to the full resolution image while "scale $n$ " represents to a coarser image where a "macro pixel" is averaged over $2^{n} \times 2^{n}$ pixels of the full resolution image. This pyramid scheme reduces the problem size and thus avoids the local minimum trap. Each images couple are correlated by the Optical Flow with Integrated kinematics within independent Blocks (OFIB). A linear system computed through the specific transformation is solved by block regardless of 
the neighbouring ones, thus, it allows identifying heterogeneous displacement fields keeping basic kinematics, namely rigid body motions, at the block scale. It can be achieved with $4 \times 4$ pixels windows ensuring a good spatial resolution.

YaDICs enables a median filter regularization at each iteration to limit uncertainties. Consider a pixels set of defined size including an aberrant value, the median filter will sort values in ascending order to identify the median value. It allows substituting the aberrant value by a consensus one regarding the neighboring values. Figure 2 shows numerical displacement field around a singularity with some added noise (normal continuous variable with a mean value of 3 and a standard deviation of 0.1) and the same field with regularizations based on Gaussian and median filters. As it can be observed, the Gaussian filter induces a "diffusion" of the singularity, this is problematic for the retained approach, as the crack shape knowledge is essential for the identification procedure. The median filter is then particularly adapted as it allows the reduction of uncertainty measurement while maintaining the discontinuity (Fig. 2).
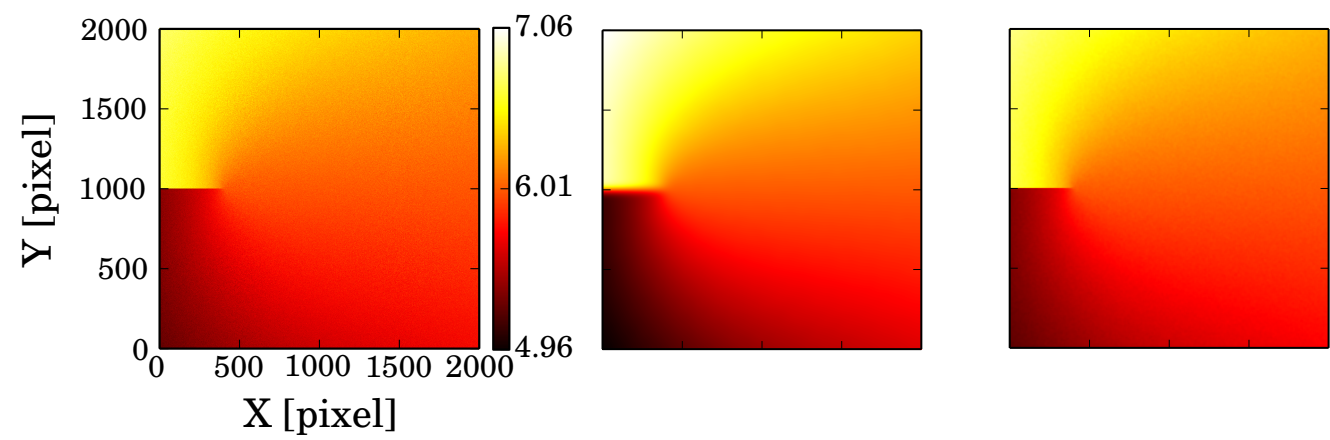

Figure 2: Numerical vertical displacements fields (in pixels) without regularization (left), with Gaussian filter (middle) and with median filter (right).

In some cases, displacements could also be searched using a decomposition on a discrete basis:

$$
\bar{u}(\bar{x})=\sum a_{n} \bar{\psi}_{n}(\bar{x})
$$

with $a_{n}$, the sought degrees of freedom and $\bar{\psi}_{n}(\bar{x})$, the shape functions.

For instance, the Finite Elements Method shape functions can be used [36] and thus suppose a continuous displacements field on the whole studied surface. The main benefit is to present the same formalism for numerical simulations so that interpolation errors can be avoided for identification [37] [38]. Furthermore, the shape functions can represent the expected behavior of displacements, called Integrated Digital Image Correlation (iDIC). This kind of approach presents a minimization that integrates measurements and identification steps. It allows saving time and avoids cumulating the errors of successive measurement and identification procedures. Models that correspond to kinematic near a crack tip such as Williams model [21] [26] could be used.

Nevertheless, the displacement field knowledge is not sufficient for the crack propagation law identification, the fields will be projected on a specific basis to assess the Stress Intensity Factor. 


\section{Williams expansion series}

Irwin [8] introduced the Stress Intensity Factor (SIF) in order to characterize the singularity at a crack tip in terms of displacements or stresses. Indeed, this parameter allows integrating the cracked specimen geometry, the length and the shape of crack and the applied load. Through this term, Williams [9] proposed the displacement in the cracked part:

$$
u(r, \theta)=\sum_{i=I, I I}[\underbrace{\sum_{n_{\min }}^{-1} \omega_{i}^{n} \phi_{i}^{n}(\theta) r^{n / 2}}_{\text {supersingular terms }}+\underbrace{\omega_{i}^{0} \phi_{i}^{0}}_{\text {translations }}+\underbrace{\omega_{i}^{1} \phi_{i}^{1}(\theta) \sqrt{r}}_{\text {singular term }}+\underbrace{\sum_{n=2}^{n_{\max }} \omega_{i}^{n} \phi_{i}^{n}(\theta) r^{n / 2}}_{\text {subsingular terms }}]
$$

with $(r, \theta)$, the polar coordinates whose origin is centered at the crack tip, $\omega_{i}^{n}$ is the sought degree of freedom and $\phi_{i}^{n}$ is the shape function for the mode $i$ of cracking and $n^{\text {th }}$ order.

In the case of confined plasticity, the Williams expansion series are valid for 2D elastic, homogeneous and isotropic medium. Depending on the $n^{\text {th }}$ order (Eq. (9)), the field will get a specific significance. The null value corresponds to the rigid body motions. The first order corresponds to analytical solutions of Westergaard [6] so that it enables to obtain the SIF ( $K_{I}$ tension part and $K_{I I}$ shearing part). The second order corresponds to the T-stress in mode $I$ and the higher orders lead to the boundary conditions for a finite medium. If the order is lower than zero, the fields are called super-singular and have no physical significance. However, the first super-singular order $(n=-1)$ is particularly useful as it allows determining the crack tip position. Indeed, the coefficient relating to this order is zero when the origin of numerical fields corresponds to the elastic equivalent crack tip [39].

Suppose that there is a gap $d x$ between the origin of constructed Williams functions and the real position of the crack front. The coefficients $\tilde{\omega}_{i}^{n}$ can be obtained:

$$
\tilde{u}=\sum_{i=I, I I} \sum_{n=-\infty}^{n=\infty} \tilde{\omega}_{i}^{n} \phi_{i}^{n}(x+d x, y)
$$

$\tilde{\omega}_{i}^{n}$ and $\omega_{i}^{n}[39]$ can be linked by proceeding in recurrence and linearisation with the equation below:

$$
\begin{gathered}
\frac{\partial \phi_{I}^{n}}{\partial x}=-\frac{n}{2} \phi_{I}^{n-2} \\
\omega_{I}^{n}=\tilde{\omega}_{I}^{n}-\frac{n+2}{2} \tilde{\omega}_{I}^{n+2} d x
\end{gathered}
$$

The first super-singular $(n=-1) \omega_{I}^{-1}$ must be equal to zero otherwise it would mean an infinite strain energy when $r$ tends to zero. With $n=-1$ and supposing $\omega_{I}^{-1}=0$, the gap $d x$ can be estimated with the ratio of $\tilde{\omega}_{I}^{-1}$ and $\tilde{\omega}_{I}^{1}$ :

$$
d x=2 \frac{\tilde{\omega}_{I}^{-1}}{\tilde{\omega}_{I}^{1}}
$$

The Direct Current Potential Drop and Digital Image Correlation were presented with the Williams series model, thus the retained strategies for the Paris law identification are investigated in the next part. 


\section{Identification approaches adopted}

\section{i DIC case}

A camera is used to acquire pictures with a definition of 2048 by 2048 pixels. It is equipped with a telecentric lens to avoid distortion and false perspectives and to reach a pixel size of $5.25 \mu \mathrm{m}$. The camera is synchronized with the force sensor in order to take pictures at the highest and lowest value of the loading at the given cycle. Each pair of pictures are correlated using YaDICs software [35] as mentioned previously. A continuous image recording avoids some transitory effects or a residual creep contribution that might occur during holding time, especially at high temperature.

The $\varepsilon_{y y}$ strain, i.e. in the loading direction which highlights the crack discontinuity, can be easily obtained from the measured displacement field and thresholded to get the crack path and tip position. From the fatigue test, which will be dealt with in the next part, the experimental strain at 75,000 cycles is represented in figure 3 .

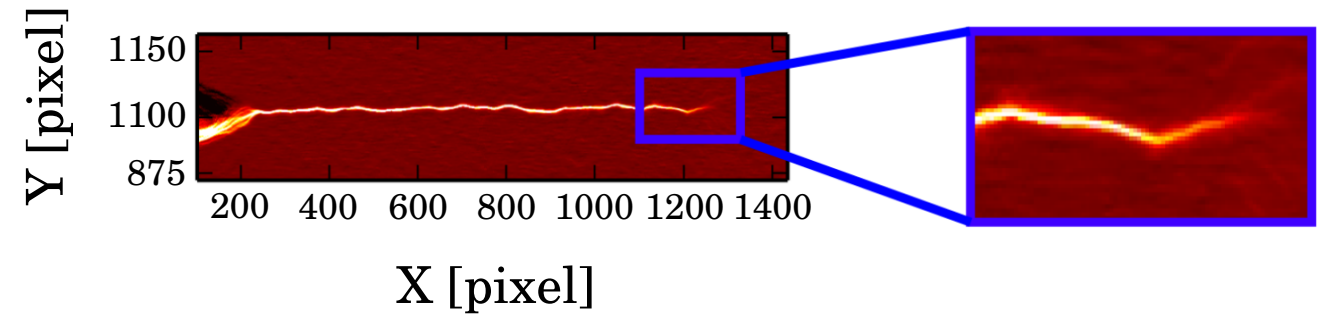

Figure 3: Experimental strain in the loading direction highlighting the fatigue crack (75,000 cycles).

The correlation residue (Eq. (14)) can also be used to accurately determine the shape of the crack [40].

$$
\varepsilon(\bar{x})=f(\bar{x})-g(\bar{x}+\bar{u}(\bar{x}))
$$

The whole crack length is always observed either at the beginning of the test, i.e. at 0 load or at the end (see Fig. 4 for the observation zone). Consequently the crack length is always measured from the notch root to the actual crack tip position of the considered cycle and rigid body motions should have no influence on the crack length measurement.

When the crack tip position is identified, numerical fields of Williams expansion series are constructed for the first two cracking modes (I in opening and II in shearing) in a range from -3 to 7 orders allowing to get sufficient accuracy [26] [41]. A mask is applied near the crack, i.e, at the location of the plastic and process zones, and all along the crack path (Fig. 4) as Williams model is based on a specific hypothesis of elasticity. The radius of the monotonic plastic zone $R_{p}$ can be estimated with Irwin's equation (plane stress hypothesis):

$$
R_{p}=\frac{1}{2 \pi}\left(\frac{K_{I}}{\sigma_{y}}\right)^{2}
$$

with $\sigma_{y}$, the yield stress of the material. 
For an expected final SIF value $K_{I}$ of $30 \mathrm{MPa} \sqrt{\mathrm{m}}$ [19] and a yield stress $\sigma_{y}$ set at $450 \mathrm{MPa}$, the plastic zone size reaches $0.7 \mathrm{~mm}$ approximately. Thus, the inner radius is set at 150 pixels (1 pixel $=5.25 \mu \mathrm{m})$.

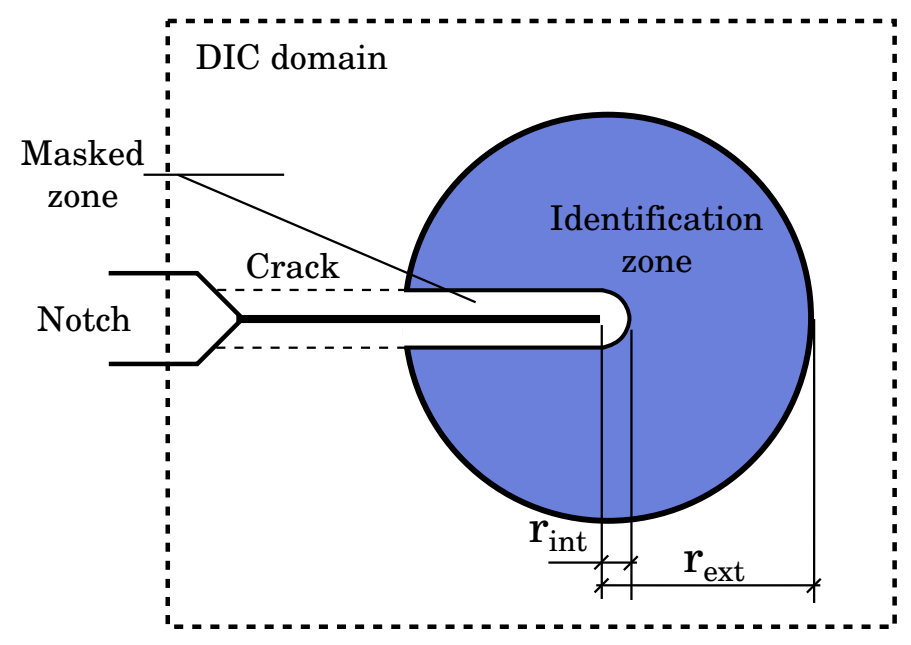

Figure 4: Projection zone with the external $r_{\text {ext }}$ and the internal radius $r_{\text {int }}$ around the crack tip.

A minimization of the difference between experimental and numerical fields is carried out through the linear least squares method. The unknown values are the degrees of freedom $\omega_{I}^{n}$ and $\omega_{I I}^{n}$ :

$$
\eta^{2}=\arg \min \left(\sum_{i=1}^{\text {pixels }}\left[\sum_{n_{\min }}^{n_{\max }}\left(\omega_{I}^{n} \phi_{i I}^{n}(\theta)+\omega_{I I}^{n} \phi_{i I I}^{n}(\theta)\right) r^{n / 2}-\left(u_{i}^{D I C}+v_{i}^{D I C}\right)\right]\right)
$$

with $u^{\text {DIC }}$ and $v^{\text {DIC }}$ respectively the horizontal and vertical measured displacements obtained with DIC.

The main difficulty of this approach is to determine with sufficient accuracy the crack tip position. Despite the positive results obtained with the thresholded strain (Fig. 3), the first super-singular order is implemented with an iterative process to get the elastic equivalent crack tip position. Thus, the threshold step is used as initialization of the position for the iterative procedure:

$$
x_{\text {tip }}^{n+1}=x_{\text {tip }}^{n}+d x
$$

where $d x$ corresponds to the shift between thresholded crack tip and the estimation with first super-singular term.

As soon as the process is completed, new shape functions are generated and the process is repeated until the shift reaches a criterion. As the crack tip location has to be accurately known for crack law identification, the criterion is set at \pm 1 pixel. 


\section{ii DCPD case}

The DCPD is an electrical tracking technique. Thus it belongs to the indirect methods whose major drawback is that a calibration is necessary in order to establish the relation between the crack size and the measured potential. Different ways to calibrate the results of the DCPD method are tested: optical crack length measurements, the Finite Element Method (FEM) and an analytical law.

- For the optical measurements method, the DIC results were used. As it was shown, the thresholded strain field highlights the crack shape (Fig. 3) and thus it benefits from DIC accuracy.

- For the FEM, the specimen is modeled with the Finite Elements code ABAQUS, using a steady-state current flow model, employing coupled thermo-electrical tetrahedral elements (DC3D4E). This assumed model involves that heat generation, time and electric transition effects are neglected. Thus, the solution to Laplace's equation, with the boundary conditions of a particular specimen geometry, has to be found:

$$
\nabla^{2}(\phi)=0
$$

with $\phi$, the steady state electrical potential.

The number of elements depends on the crack size and can reach 13,000 tetrahedra. The measured potential areas are modeled with rectangle surfaces positioned symmetrically from either side of the crack. The current injections are also represented with rectangular surfaces that correspond to the boundary conditions and the current value is set at $2 \mathrm{~A}$. With these hypothesis, the only necessary material parameter is the electrical conductivity set at $1680 \mathrm{~S} / \mathrm{mm}[42]$.

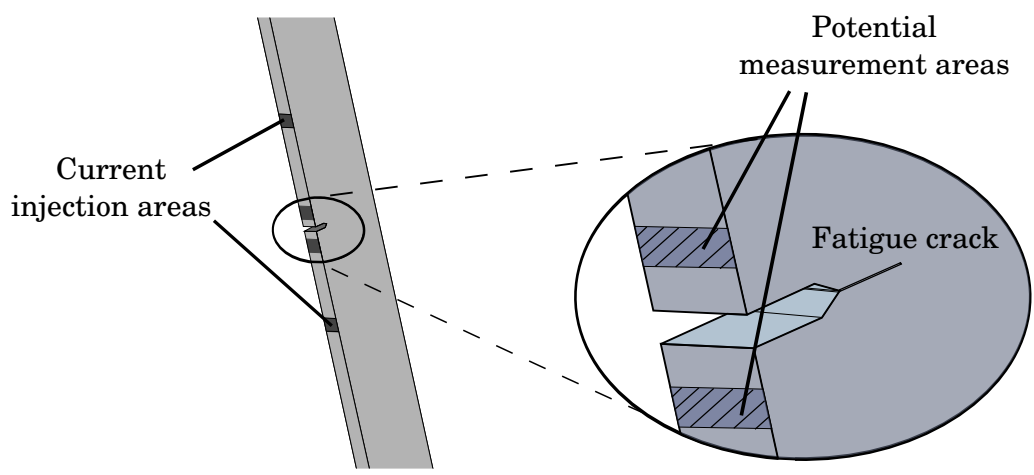

Figure 5: Definition of current injection leads and potential measurement areas in the FEM case.

Calculations are done with different crack sizes and thus a calibration law can be identified by fitting a polynomial function for example:

$$
a=6.32 \times 10^{-3}\left(\frac{V}{V_{0}}\right)^{3}-1.78 \times 10^{-2}\left(\frac{V}{V_{0}}\right)^{2}+2.73 \times 10^{-2}\left(\frac{V}{V_{0}}\right)-1.14 \times 10^{-2}
$$

Figure 6 shows the disturbed potential field due to the crack and the current distribution near the crack for a $5.4 \mathrm{~mm}$ long crack. 

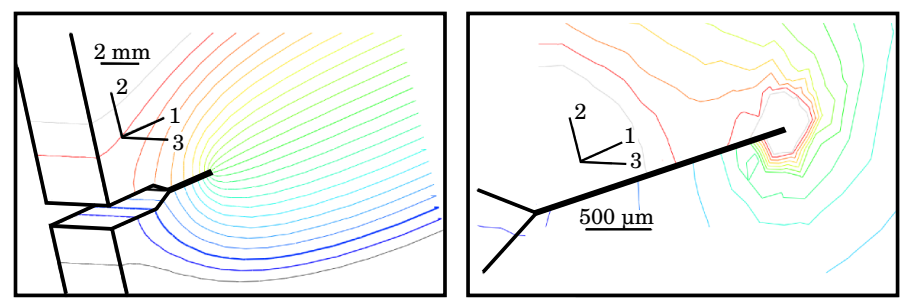

Figure 6: Electrical potential and the current density for a FEM calibration for a specific length of $5.4 \mathrm{~mm}$.

- The crack length can also be expressed with the potential difference through the analytical Johnson's formula [32]. This law is given for a through-crack:

$$
a=\frac{2 W}{\pi} \cos ^{-1}\left[\frac{\cos \left(\frac{\pi y}{2 W}\right)}{\cosh \left(\frac{V}{V_{0}} \cosh ^{-1}\left[\frac{\cosh \left(\frac{\pi y}{2 W}\right)}{\cos \left(\frac{\pi a_{0}}{2 W}\right)}\right]\right)}\right]
$$

with $V$ the measured potential that is related to the crack length $a$. The length $W$ is the specimen width, $y$ is the distance from the potential probe to the crack and $V_{0}$ and $a_{0}$, are the initial potential and crack length.

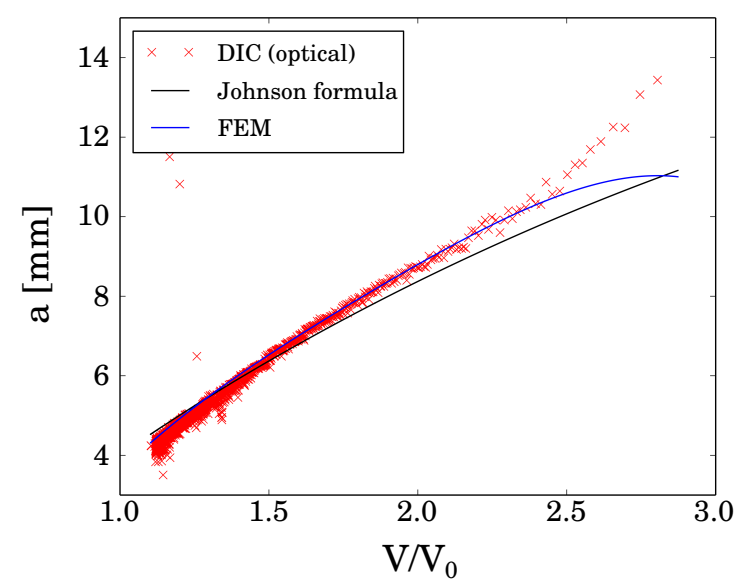

Figure 7: Optical, analytical and FEM calibration curves for a SENT specimen.

In practice, calibration curves are given in the form $V / V_{0}$ where $V_{0}$ is the reference potential drop across the initial crack. Therefore, calibration curves become independent of material properties and of input current magnitude and will depend only on the location of the current inputs and potential measurement leads [43]. The crack length versus normalized potentials according to the three methods are plotted in figure 7. Results appear to be very close especially for a limited crack length. However, the Johnson formula tends to deviate from the two other calibrations for crack length exceeding $7 \mathrm{~mm}$, which corresponds to an $a / W$ ratio close to 0.4 . It conducts to an underestimation of the crack length. It could observed that some points from DIC analysis reached almost $12 \mathrm{~mm}$ whereas the physical crack length is around $5 \mathrm{~mm}$. This analysis artefact, 
very limited at the beginning of the test, is due to a too much severe level of threshold at the crack tip.

In order to compare DIC and DCPD methods, a calibration independent of the optical measurements is preferred. The Johnson's formula has been widely used and improved [12] but remains hard to apply. Furthermore, it needs to be extremely accurate on the probes location knowledge, particularly with short cracks [12]. Hence, the finite elements method is retained permitting to take into account the cracked geometry and probes locations for a plane crack propagation. For long cracks, the errors in terms of location of the probes or current leads have a limited impact on the accuracy of the method.

After calibration, electrical measurements give the crack length but no information about the SIF values. Analytical formulas are used to link $K_{I}$ and $a$ [15]:

$$
K_{I}=Y \frac{F}{B W} \sqrt{\pi a}
$$

where $Y$ is a function to correct the finite size effects, $B$ and $W$ are the thickness and the specimen width. The corrective function can be expressed as:

$$
Y=\sum_{i=1}^{5} k_{i}\left(\frac{a}{W}\right)^{(2 i-1) / 2}
$$

For a specific geometry, $k_{i}$ coefficients are defined in some handbooks [14]. For a throughcrack, the corrective function corresponds to:

$$
Y=1.99\left(\frac{a}{W}\right)^{1 / 2}-0.41\left(\frac{a}{W}\right)^{3 / 2}+18.7\left(\frac{a}{W}\right)^{5 / 2}-38.48\left(\frac{a}{W}\right)^{7 / 2}+53.85\left(\frac{a}{W}\right)^{9 / 2}
$$

\section{Application to A FAtigue test With CONSTANT MAXIMUM LOAD}

The two previous approaches are applied in the case of a crack propagation test with a constant maximum load value to assess the crack tip location $a$ and the stress intensity factor $K_{I}$. These values are usual inputs of the Paris' fatigue crack propagation law. Indeed, this law is expressed as a function of the crack growth rate versus SIF range. Consequently, its expression without propagation threshold is defined as:

$$
d a / d N=C(\Delta K)^{m}
$$

\section{Test conditions}

Because of the shape and the small dimensions of the raw material, the chosen specimen geometry is the Single Edge Notch Tension (SENT) type. The width of the specimen is $W=18 \mathrm{~mm}$ and its thickness is $B=3.8 \mathrm{~mm}$. Its length is $L=140 \mathrm{~mm}$ with a notch $a_{0}$ of $3.4 \mathrm{~mm}$. The specimen is subject to a sinusoidal loading profile with a constant maximum value of $7 \mathrm{kN}$. The load ratio is 0.1 and the frequency is set at $2 \mathrm{~Hz}$. The specimen (to which spray paint is applied as a speckle pattern) is recorded with a synchronized camera to take pictures at maximum and minimum load values every 50 cycles (Fig. 8). For the DCPD method, a generator applies a constant intensity current of $2 \mathrm{~A}$ through the specimen and the potential difference across the crack is followed with a nano-voltmeter at the maximum load value of each cycle, i.e. at $7 \mathrm{kN}$. 
The device used is an AGILENT 34420A voltmeter which presents high sensitivity at $100 \mathrm{pV}$. The specimen is electrically insulated from the grips of the testing machine by using elastomeric plates, which are placed directly between the cracked specimen and the grips.
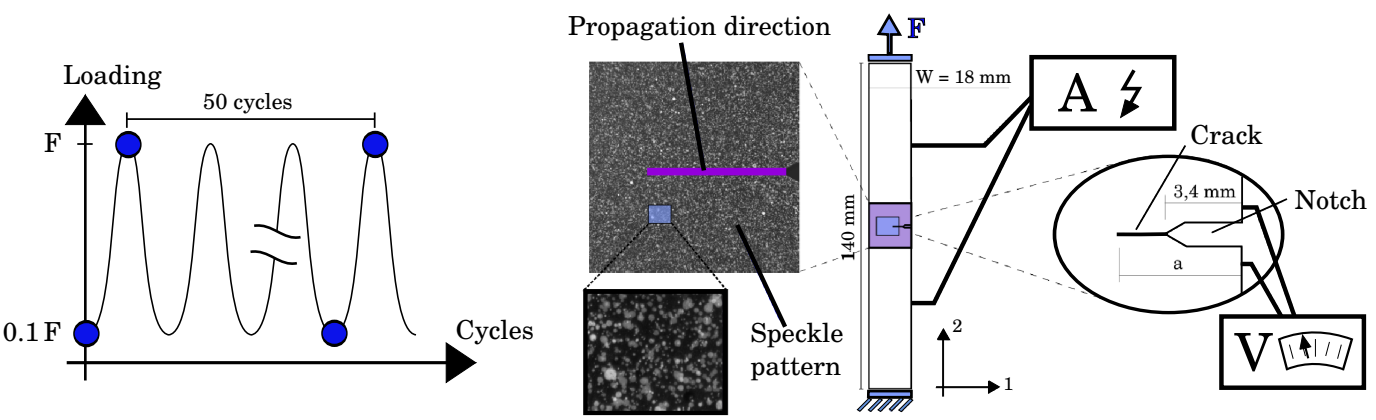

Figure 8: On the left the loading profile considered and on the right, the speckle pattern with the studied zone of the specimen and inputs/outputs for DCPD method.

The specimen is pre-cracked to overcome the notch influence. A low load amplitude is applied to minimize plasticity during this step. The retained experimental procedure consists in modifying the loading path in order to limit the SIF increase [30] [22]. This procedure corresponds to a load shedding approach allowed by DCPD method. Here, the specimen was pre-cracked at constant stress intensity of $K_{I}^{\max }=12 \mathrm{MPa} \sqrt{\mathrm{m}}$, with a sinusoidal cyclic loading at a load ratio of 0.1 , to generate a one millimetre long sharp pre-crack.

The studied material is a Spheroidal Graphite (SG) cast iron enriched in silicon and molybdenum, sampled from foundry bars of length $200 \mathrm{~mm}$ and diameter $20 \mathrm{~mm}$ obtained by gravity casting. Its heterogeneous microstructure consists of a ferritic matrix presenting well distributed graphite inclusions. The chemical composition of this ductile cast iron is shown in Tab. 1.

\begin{tabular}{ccccc} 
C & Si & Mo & Mn & P \\
\hline 3.4 & 3.89 & 0.57 & 0.15 & 0.025 \\
\hline
\end{tabular}

Table 1: Chemical composition of the studied cast iron (weight \%).

The graphite nodules are well distributed in the material as proved by X-ray micro-tomography characterization. Thus, the ductile cast iron should present an isotropic electrical behaviour easy to handle with the DCPD method as confirmed by its use to monitor crack lengths [44].

The fracture of the specimen is observed at around 80,000 cycles after cycling the pre-cracked specimen at a constant maximum load value of $7 \mathrm{kN}$.

\section{Crack length evaluation}

The first step is the crack length estimation. It is computed for DIC method by thresholding the discontinuity through the gradient of the measured displacement (Fig. 3), then for the DCPD method by using a specific calibration law (Eq. 19). First, to refine the study, some parameters are tested especially for DIC method. Then, at several steps of the test, the two modalities are compared. 


\section{i Impact of the windows size and the Williams terms}

DIC calculations are carried out with various window sizes in order to appreciate its impact on the crack length estimation. The study considers DIC calculations with windows of $4 \times 4,8 \times 8$ and $16 \times 16$ pixels at major steps of the cracking test.
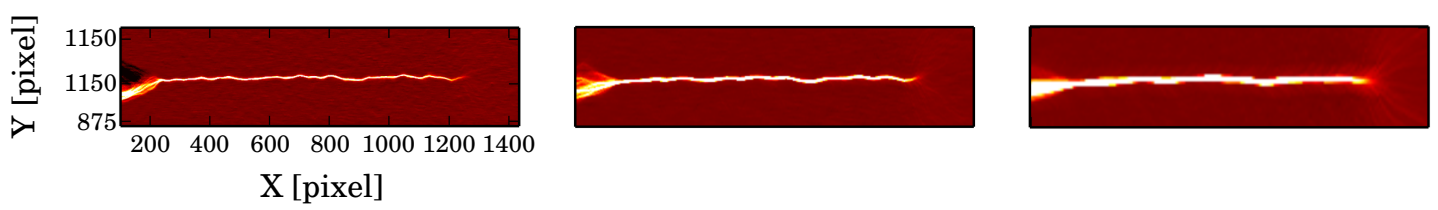

Figure 9: The crack shapes for different windows sizes: $4 \times 4$ (left), $8 \times 8$ (middle) and $16 \times 16$ pixels (right).

In figure 9, the crack shapes are shown for the cycle 75,000. As it can be observed, for $4 \times 4$ pixels window size, the crack shape is perfectly defined, enabling local crack tilting observations. The crack shape is less well-defined with larger windows, affecting the crack tip location measurements. Figure 10 shows the crack length over cycles for the different windows sizes. Indeed, a relative overestimation is observed in the cases of the $8 \times 8$ and $16 \times 16$ windows, up to a maximum of $0.2 \mathrm{~mm}$ as compared to the $4 \times 4$ sizes.

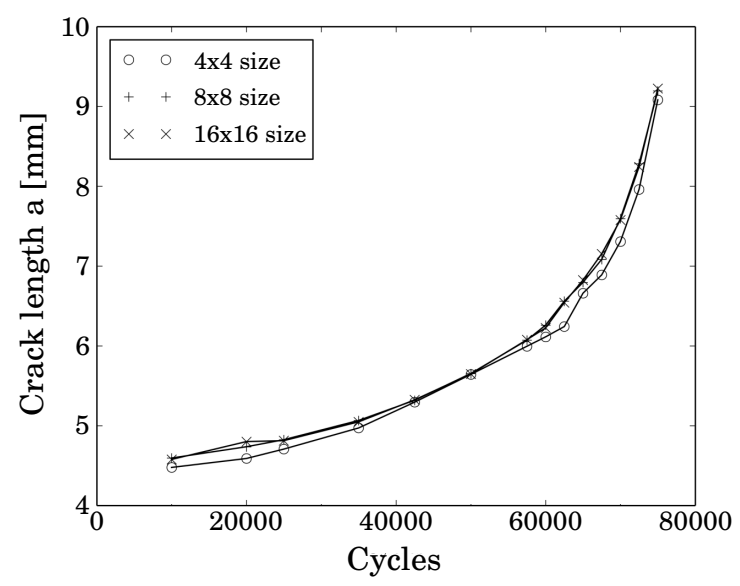

Figure 10: The crack length versus cycles for three different windows sizes of DIC calculations.

In the literature, the first super-singular mode of the Williams expansion series is commonly used, a priori to obtain a better estimation of the crack tip location (Eq. 13). Therefore, the developed approach can be compared with one including the first super-singular term in an iterative procedure. The results in figure 11 show that the crack tip locations ("1 to 7 " data serie) are close to the equivalent elastic tip positions calculated with the $-1^{\text {st }}$ term of the Williams series, i.e. "- 1 to 7 " and "-3 to 7" data series. 
The first super-singular term is not necessary to estimate the crack length as observed in figure 9 , the fields with $4 \times 4$ pixels size provide a good resolution to estimate the crack length. Moreover, the super-singular terms can conduct to deviations from only singular and subsingular terms (Fig. 11). Therefore, the method considering only singular and subsingular Williams terms is chosen to ensure some robustness.

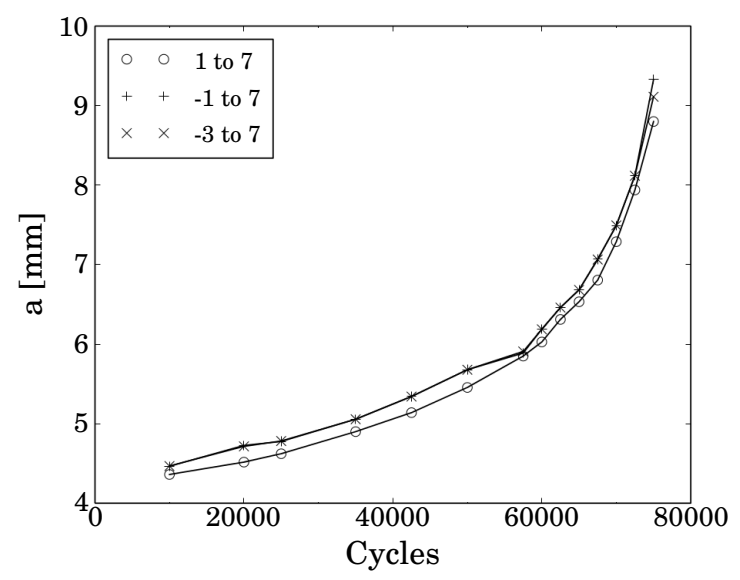

Figure 11: The crack length versus cycles obtained with three different ranges of Williams terms.

\section{ii DIC and DCPD comparisons}

After the optimum parameters definition for DIC, this method and the DCPD's can be compared in terms of crack length estimations. Figure 12 represents the measured crack length over cycles with some magnifications at different specific times of the test.

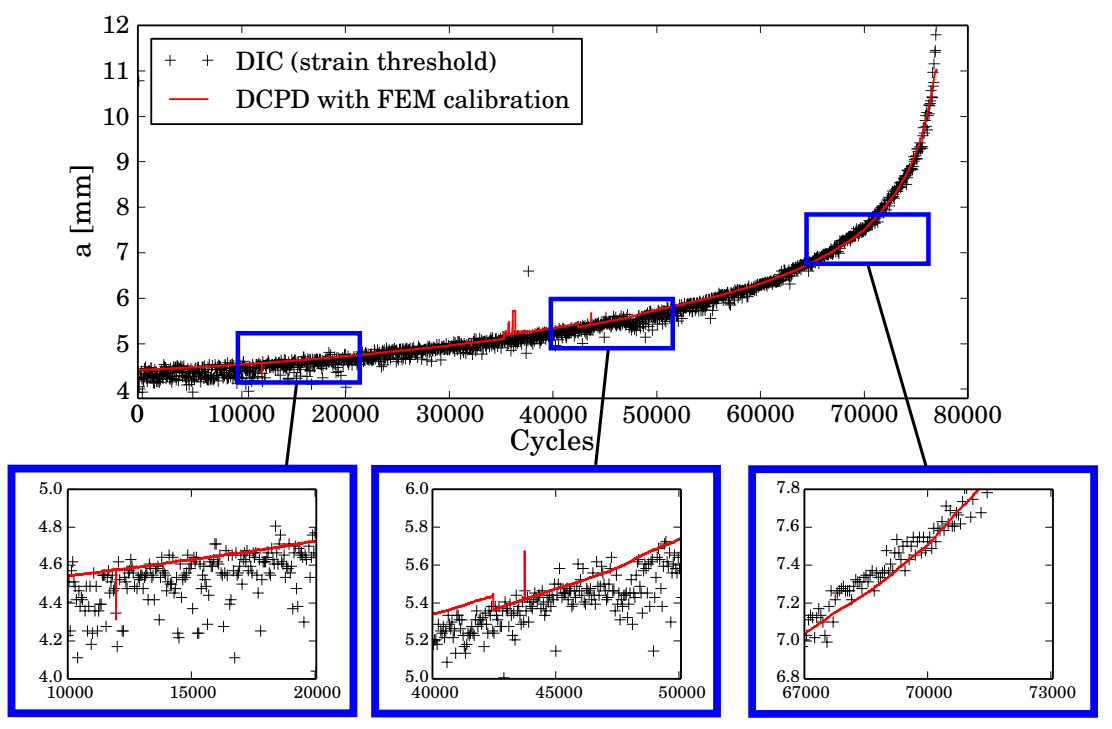

Figure 12: The measured crack length versus number of cycles for the two retained approaches. 
Results present a good agreement during all the test. Some noise is present due to the signal acquisition, leading to an alteration of the maximum and the minimum of the load. Data have to be filtered. The uncertainty level decreases during the test, owing to a more marked opening of the crack. The dispersion of the measured values is close to $0.4 \mathrm{~mm}$ all along the first part of the propagation to reach a nearly $0.1 \mathrm{~mm}$ value at the end. It is relatively similar to the crack length resolution of the DCPD method of nearly $50 \mu \mathrm{m}$ [30].

Moreover, the crack path, which is highlighted with strain fields in figure 9, is rather characterized with a tortuosity at a micro-scale (Fig. 13).

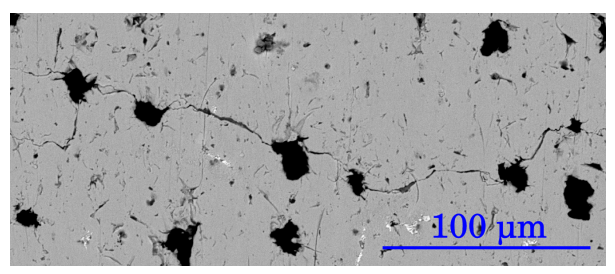

Figure 13: Crack path of a fatigue crack highlighting tilts due to the graphite presence (the crack grows from left to right) with a $\Delta K$ close to $20 \mathrm{MPa} \sqrt{\mathrm{m}}$ (close to 70,000 cycles).

With Scanning Electron Microscope (SEM) observations of the fracture surface, a marked topography was observed, validating the micro tortuosity seen at the surface. The crack presents a global tri-dimensional aspect, consequently, the DIC surface measurement can be impacted with this kind of "artefact". Indeed, the DCPD method is globally tri-dimensional as the measurement integrates the specimen width, unlike the DIC, which is a surface measurement. However for long crack study, the tri-dimensional aspect of the crack has no impact on the crack length estimation. The measurements can also be perturbed with some physical phenomenon; it is particularly the case of the resistivity that evolves with the plasticity. When the plasticity is well confined, it does not affect the measurement, whereas for a long crack, the plasticity becomes more pronounced (at constant maximum load) and shifts can appear. Indeed, Doremus et al. [12] recorded the potential drop during a tensile test and highlighted clearly an important resistivity increase. But this resistivity evolution must be limited during the test as it was not observed by means of the comparison of DIC and DCPD methods.

\section{Stress intensity factor evaluation}

First, it is proposed to perform a comparative study to appreciate the identification results from different DIC calculations by modifying the windows size and the Williams terms number, similarly to what was done for the crack length evaluation.

\section{i Impact of windows size}

As shown before, the use of $4 \times 4$ pixels windows highlights the crack shape in an efficient manner. But, the main drawback is to obtain more noisy data, despite the median filter use, that could affect the projection quality on Williams series. Therefore, the residue is used to appreciate the results accuracy. It is obtained by subtracting the reference image to the deformed image corrected with identified fields : $f(\bar{x})-g(\bar{x}+\bar{u}(\bar{x}))$. 
In figure 14, only a low impact is observed on the residue until 50,500 cycles with a measured shift between $16 \times 16$ and $4 \times 4$ results close to 0.01 . Beyond this point, the gaps between $16 \times 16$ and $4 \times 4$ results can be five times larger as at the end of the test. For the SIFs identification, the best compromise seems to be the use of $8 \times 8$ pixels window size, as the residue values are close to those of $16 \times 16$.

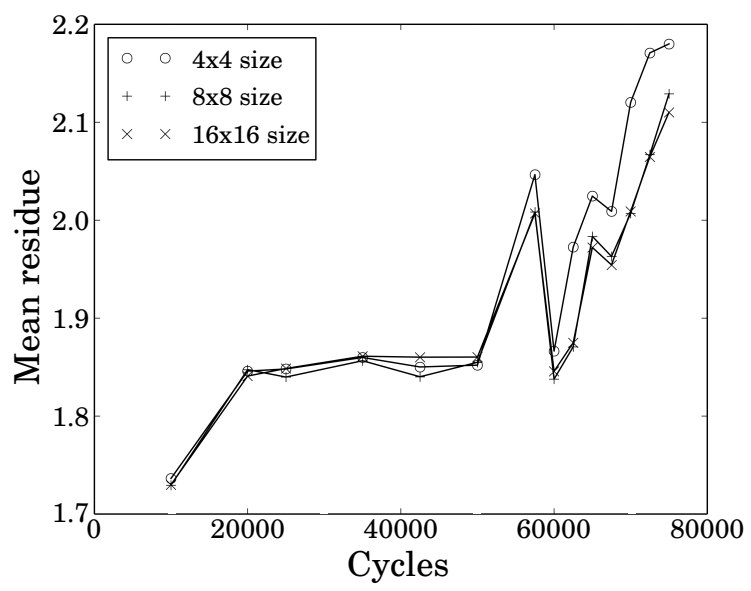

Figure 14: The mean grey residues $|f(\bar{x})-g(\bar{x}+\bar{u}(\bar{x}))|$ versus cycles for three different windows size of DIC.

\section{ii Impact of Williams terms number}

To obtain an accurate description of the displacement field around the crack tip, the supersingular terms should be taken into account a priori. As it was shown previously, Williams series orders often range from -3 to 7 and it seems to be sufficient to describe the observed zone. The study was carried out with $-3 \leqslant n_{\min } \leqslant 1$ and $1 \leqslant n_{\max } \leqslant 8$ for different crack lengths recorded during the fatigue test.
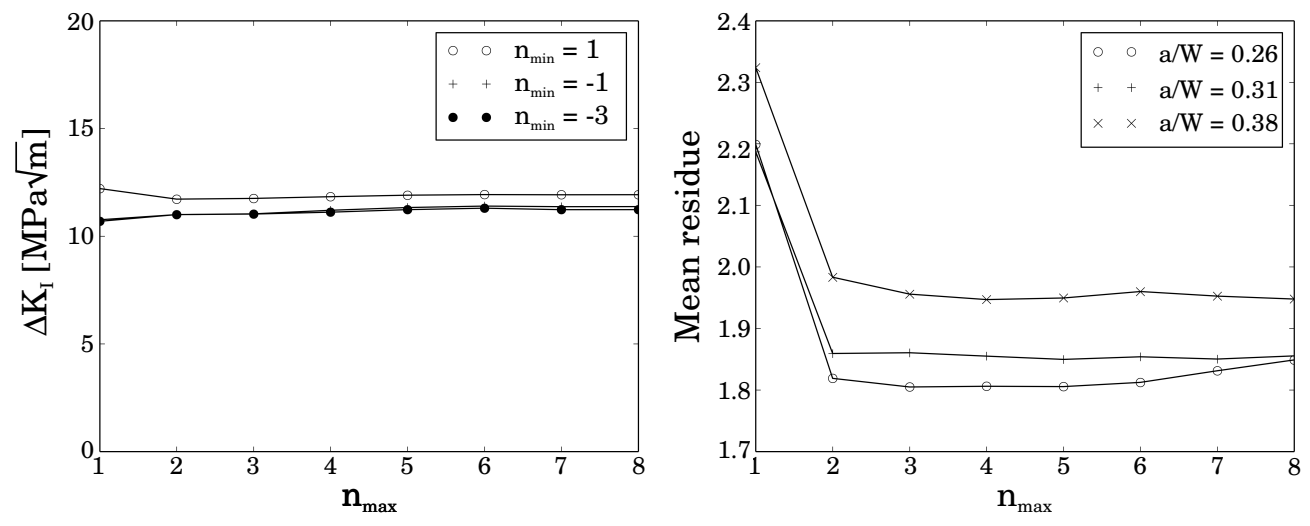

Figure 15: On the left, identified SIF versus $n_{\text {max }}$ for different ranges of Williams terms and on the right, the mean grey level residues versus $n_{\max }$ for different crack lengths without super-singular terms, i.e. $n_{\min }=1$. 
As shown in figure 15, few terms seem to be sufficient to obtain a stable value of $K_{I}$ whatever the crack length. The super-singulars terms are not essential to obtain a stable value of $K_{I}$, the observed gaps are limited, i.e. less than $1 \mathrm{MPa} \sqrt{\mathrm{m}}$. Moreover, the duration of the calculation for a range from 1 to 7 is four times faster than when super-singular terms are considered due to the iterative aspect of the procedure, requiring the re-formulation of the shape functions.

The high order terms allow correcting the finite size effects. These effects become more important when $a$ increases, because the distance between the crack tip and the free surface downstream of the crack decreases. As a consequence, for long cracks, the displacement fields are more sensitive to the edge effects and higher order terms have to be added in the displacement field decomposition. That is why, the mean residues are larger for higher $a / W$ ratios (Fig. 15). For the next parts, the Williams series from 1 to 7 are considered.

\section{iii Impact of mask zone size}

To take into account the elasticity hypothesis considered in the Williams model, displacement values close to the crack tip have to be masked in order to limit the plasticity impact on the identification. In addition, values close to the boundary conditions have to be neglected too to limit the maximum Williams order required and to find an optimum between accuracy and speed of calculations. A ring is considered (Fig. 4) as an identification zone with an inner radius and a variable external radius. As it was shown before, the inner radius, set at 150 pixels $(1$ pixel $=5.25$ $\mu \mathrm{m}$ ), is obtained with Irwin's equation (Eq. 15). The identification points number is modified with the variable external radius. The analysis of the influence of the external radius parameter was carried out for different images corresponding to different crack size $(a / W)$.

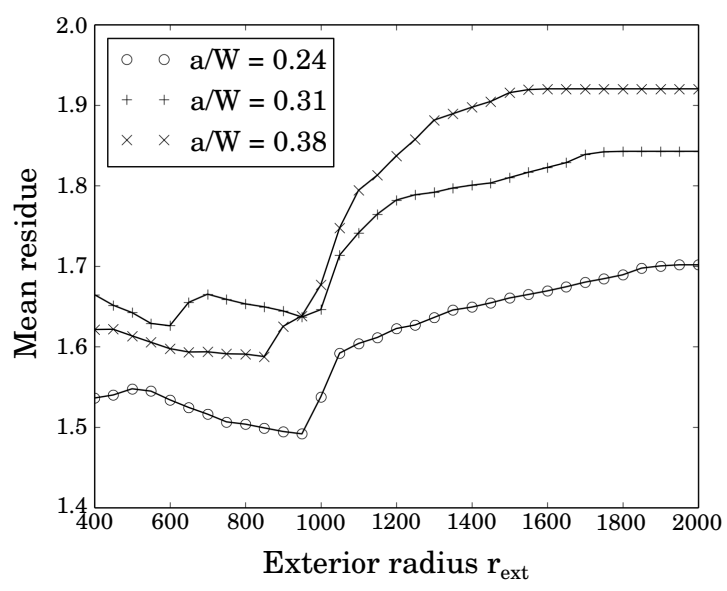

Figure 16: The mean residue versus the external radius for different ratios of the crack length.

The figure 16 shows the residues evolution as function of the external radius for different crack lengths. Two major regimes can be distinguished, corresponding to the K-dominance area driven by the SIF and a larger domain than this latter, where the boundary conditions are more pronounced. Indeed, the mean residues decrease at first and then increase after passing through a global minimum. When the mask size is not enough large, the solution stability deviates significantly and when it is too large, the more important residue can be explained by a lack of information. As a conclusion, the residue minimum is globally included between nearly 800 and 1000 pixels and for the next parts, a 800 pixels radius size is retained. 


\section{iv DIC versus iDIC}

DIC can be achieved with specific shape functions corresponding to the expected kinematic near a singularity such as Williams expansions series. It allows limiting the noise because it gathers the fields calculation and identification steps [45]. This method is called Integrated DIC (iDIC). DIC and iDIC are compared at different crack positions to appreciate the specific contribution of an integrated approach.

Crack lengths acquired from thresholded strains in the loading direction are retained. Furthermore, to improve the calculations efficiency, degrees of freedom are initialized with the values obtained by the chosen projective method.

The residue fields, plotted in figure 17, are relatively low for each method corresponding to less than $1 \%$ of the dynamic range of grey levels. It validates the good measurement of the displacement fields and the validity of each approach, with residue values globally stable during all the test. However, the integrated correlation presents better mean residues than the projective DIC, especially until 60,000 cycles. Beyond this, the integrated correlation residues increase to get closer to the stable value of the projective DIC. Contrary to Mathieu et al. [26], the mean level depends on the cycle number for iDIC, though its increase is limited.
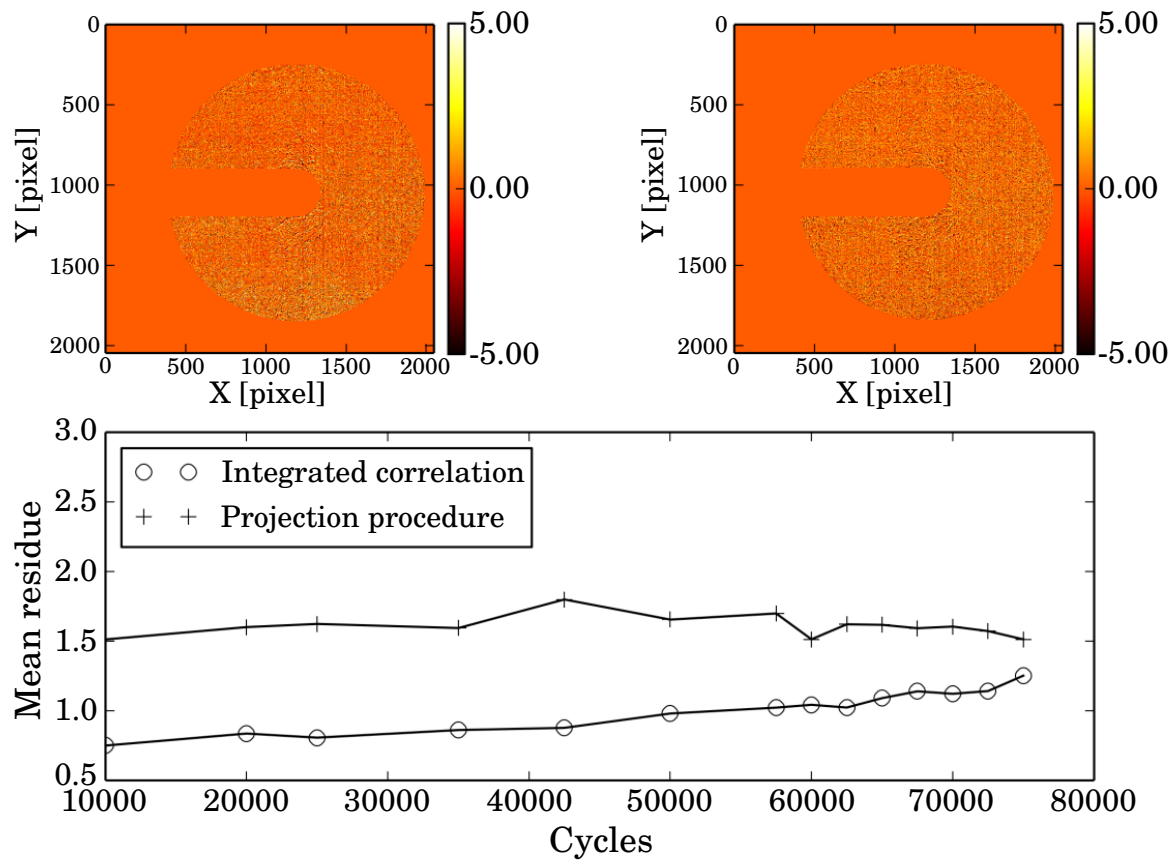

Figure 17: Evolution of mean grey level residues with a projective and an integrated approaches and residue fields for each method at 65,000 cycle (left and right respectively).

\section{v DIC/iDIC procedures versus DCPD}

Figure 18 shows the stress intensity factor as a function of the measured crack tip position. Close results can be appreciated between DIC and DCPD methods, particularly at the beginning of the test. For this latter technique, SIF corresponds to the analytical solution (Eq. 21). Above $9 \mathrm{~mm}$, the DCPD approach overestimates the SIF, whereas iDIC and DIC approaches remain accurate. 


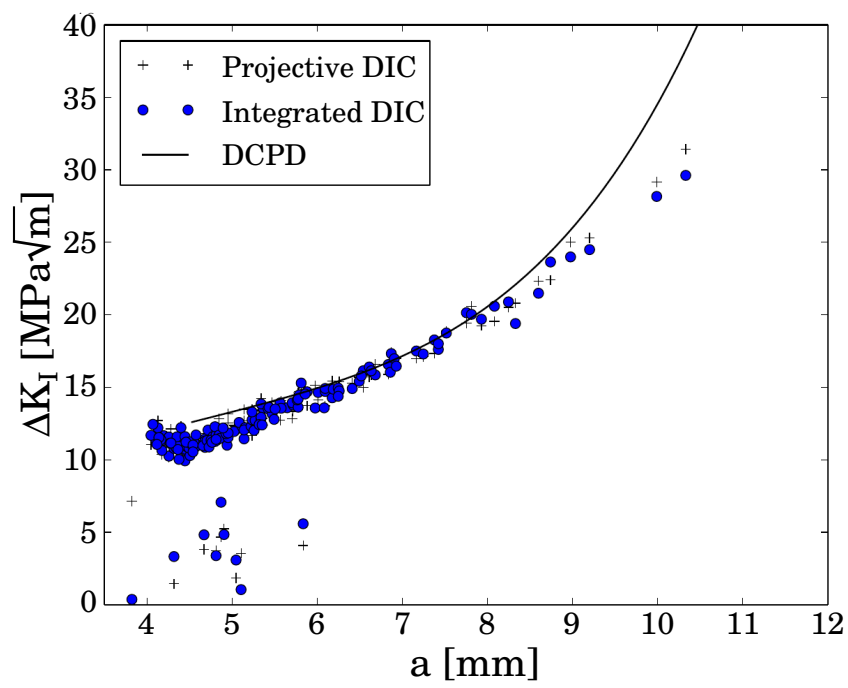

Figure 18: The SIF range versus crack length for each modality.

This agreement shows the accuracy of DIC/iDIC calculations and is considered as a validation of the procedures. The three techniques show a good agreement for measured SIFs between 12 and $25 \mathrm{MPa} \sqrt{\mathrm{m}}$. The iDIC added value seems to be negligible. This can be explained by the good results of classic DIC using a median filter regularization. To conclude, despite noisy data, DIC and iDIC both allow a wealth of information independent of the specimen's geometry, which is not the case with DCPD method.

\section{Paris law identification}

Finally, the reliable values of the crack tip location and of the SIF allow the identification of the fatigue crack propagation law using the above approaches. The projective approach is retained and undertaken with:

- 4x4 and 8x8 pixels DIC window sizes for the crack length and SIF identifications, respectively.

- Williams orders range from 1 to 7 allowing to deal with the last stages of crack propagation where boundary conditions are more pronounced.

- an identification zone with an inner radius set at 150 pixels and an external radius of 800 pixels.

A direct fit between the measured SIF range $\Delta K_{I}$ and the crack growth rate $d a / d N$ is not conceivable because of noisy data (Fig. 18). To improve results, the crack length is fitted with polynomial functions, two functions are used in order to avoid underestimating the values at the end of the test. Figure 19 shows the comparison of the experimental crack growth rates measured in this work with projective approach with some literature data on the same material. 


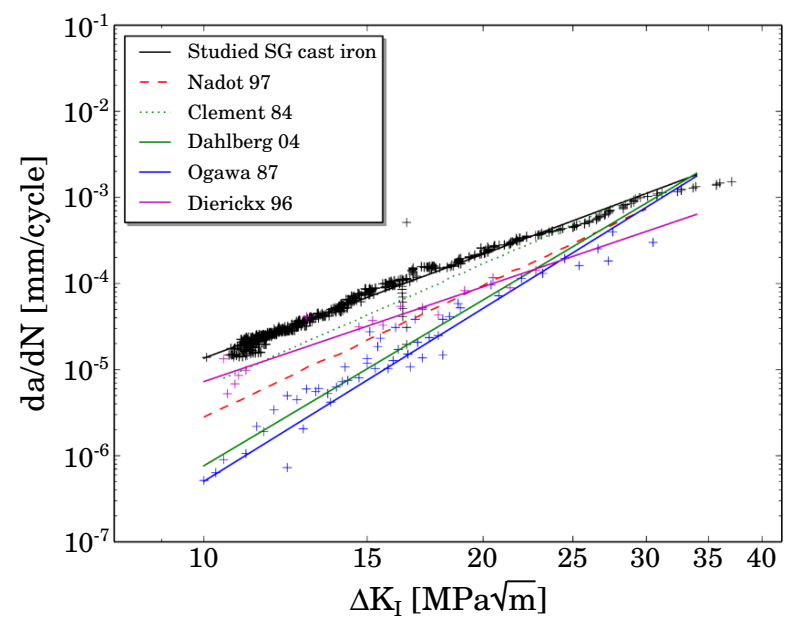

Figure 19: Crack growth rate as a function of the variation of the stress intensity factor for the studied SG cast iron and literature data [46] [47] [48] [49] [50] at R-ratio of 0.1.

The observed behavior is similar to the literature values. The projective approach is full of information, owing to the pictures taken every 50 cycles, unlike many authors' results. Paris parameters are determined for each technique and are given in Tab. 2.

\begin{tabular}{ccc} 
Parameters & $\boldsymbol{C}$ & $\boldsymbol{m}$ \\
\hline Projective DIC & $10^{-8.9}$ & 4.03 \\
\hline Integrated DIC & $10^{-8.83}$ & 4.01 \\
\hline DCPD & $10^{-8.87}$ & 4.07 \\
\hline Dierickx [50] & $10^{-9.0}$ & 3.81 \\
\hline
\end{tabular}

Table 2: Identified parameters of Paris law and comparison with literature values [50].

A good agreement is observed between each modality. Despite the better fit quality for iDIC, very similar results are obtained in terms of propagation parameters. The toughness of SG cast iron is close to $35 \mathrm{MPa} \sqrt{\mathrm{m}}$. At low velocity, a decrease is observed and can be considered as the propagation threshold with value of $8-10 \mathrm{MPa} \sqrt{\mathrm{m}}$. Nevertheless, a test with modification of loading path would be required to accurately estimate the threshold.

\section{Plasticity at the crack tip}

For a constant maximum load test, the propagation velocity becomes important because of a quick increase of SIF and consequently an uncontrolled plastic zone size (Eq. 15). It is proposed to appreciate this phenomenon by considering the model deviation through the identification residues. Indeed, the difference between experimental and numerical fields brings some information due to the fact that the Williams series are based on an elasticity hypothesis. The residues reached high values near the crack, highlighting the deviation between model and experiment. As shown by the different propagation steps in figure 20, a gradual increase appears during the test, corresponding to increasingly important plasticity effects. 
With an appropriate threshold, the plasticity can be separated from elastic part and quantified. Otsu's method [51] is retained as it is particularly adapted to automate the procedure using the histogram shape.
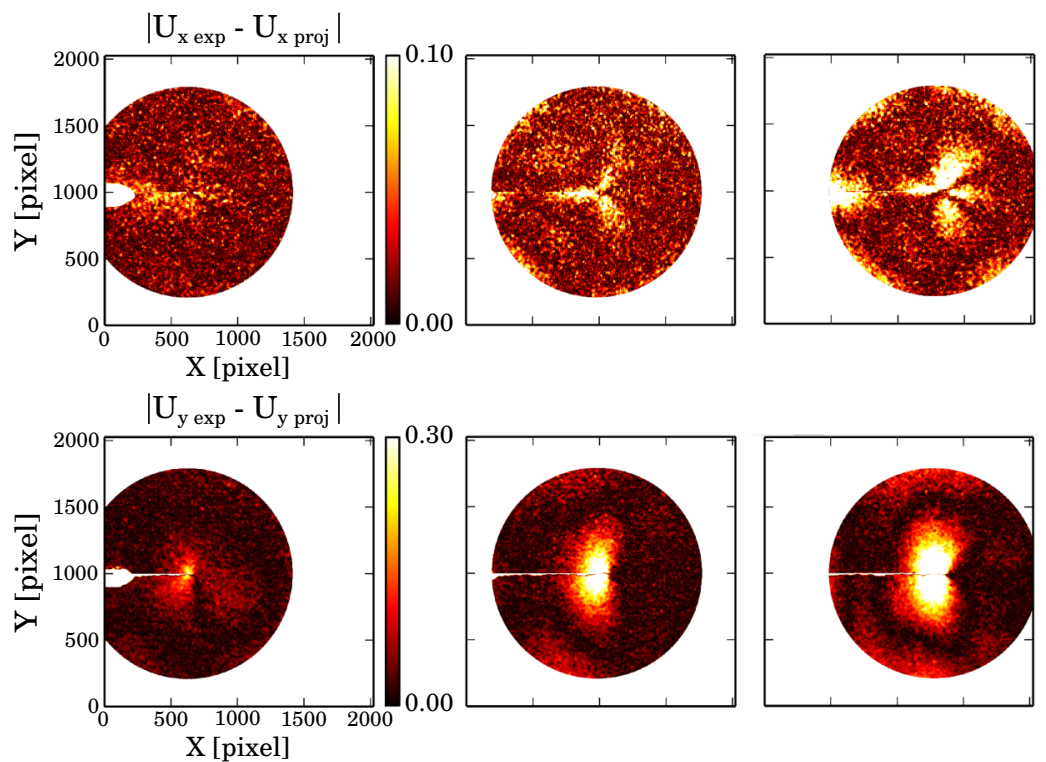

Figure 20: At the top, the horizontal residues and at the bottom, the vertical residues for crack lengths of 6.1, 8.9 and $10.9 \mathrm{~mm}$.

The plastic zone size corresponds to the shift between the crack tip location estimated from the strain field and the farthest thresholded point location in the residue.

Finally, Williams expansion series provide a specific information on this kind of non-linearities. Indeed, the third super-singular term is homogeneous to a length scale of the plastic zone [5]:

$$
R_{p} \propto \sqrt{-8 \frac{\omega_{I}^{-3}}{\omega_{I}^{-1}}}
$$

with $\omega_{I}^{i}$ the amplitudes of $i^{\text {th }}$ order in mode $I$.

These estimations are represented in figure 21 all along the crack propagation. Irwin's analytic law and the model deviation threshold present very close results, highlighting the fact that the threshold procedure is particularly reliable. As in Mathieu et al. [26], the projective approach does not seem to access to a meaningful measurement through the third super-singular term.

Only projective approach enables the plasticity observations as displacements measurements and identification procedures are separated unlike iDIC that gathers these two steps. Finally, the DCPD method does not deal with the inelastic phenomenon. 


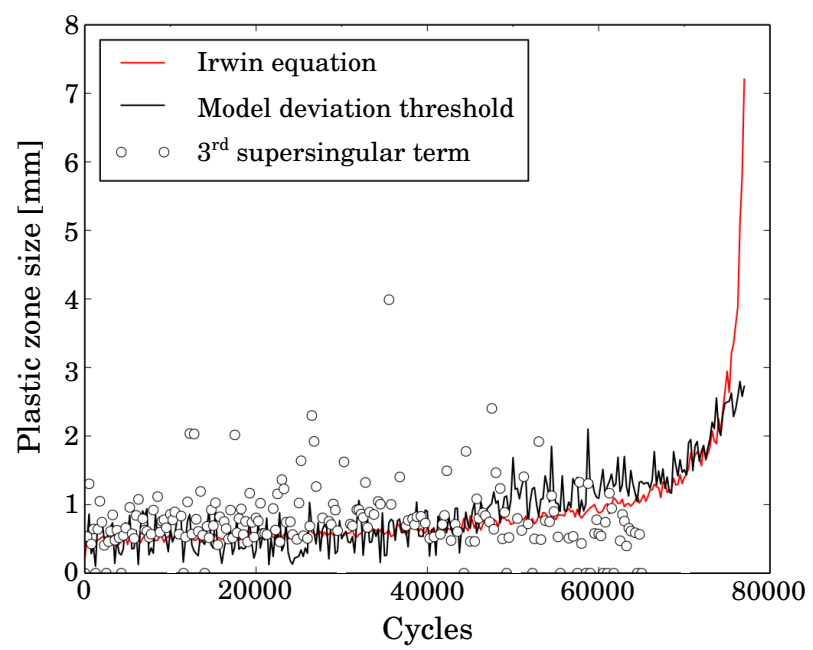

Figure 21: Plastic zone sizes obtained with Irwin's equation, the threshold of model deviation and the third super-singular term versus number of cycles.

\section{CONCLUSIONS}

Two techniques were introduced as the Digital Image Correlation and the Direct Current Potential Drop methods, in order to characterize long crack behavior and especially to identify the Paris' law. This identification requires an estimation of the crack length and the Stress Intensity Factor. These methods present some advantages, but also limitations:

- Measuring process - The DCPD method is particularly interesting in a closed environment without optical access. The measurement is continuous, obtained for each cycle and allows an access in real-time to $a$ and $K_{I}$ through appropriate calibrations and analytical laws. DIC allows a more difficult measurement for each cycle because of technical limits (images storage, post-treatment of the pictures...). Nevertheless, these limits begin to be overcome by GPU calculations. Imaging each $50-100$ cycles allows a priori to yield enough information quantity for a fatigue test.

- Measurement accuracy - The literature emphasized a DCPD resolution of nearly $50 \mu \mathrm{m}$ for crack detection whereas with DIC, it evolves from 100 to $400 \mu \mathrm{m}$. The integrated approach also presents a lower residue than the projective one. Nevertheless, all identified Paris values are close to those of the literature.

- Multiaxiality - DIC can deal with multiaxial cases for a straight propagation to obtain the SIFs in the three cracking modes (with stereo-DIC for the out-of-plane shearing term) contrary to the DCPD, limited to the opening cracking mode. The DIC procedures are based on an analytical model describing the kinematic at the crack tip independently of the specimen geometry. Finally, the projective approach can be extended to cracks with bifurcations.

- Plasticity - The DCPD method presents a more intrinsic elastic behavior than DIC. Indeed, the first one is based on analytical law supposing an elastic medium. Nevertheless, the plasticity is unavoidable with electrical resistivity modifications conducting to more shifts on the SIF identifications at the end of the propagation. DIC permits to mask displace- 
ment values impacted by the plasticity at the crack tip, thus constraining its influence on identification. Moreover, DIC deals with plasticity through the identification residues. A progressive increase was shown, highlighting the importance of such phenomenon at the end of the fatigue crack propagation.

Generally speaking, the obtained results for each method are very close in the confined plasticity case. It requires limited equipment such as a camera and DIC software/a generator and an accurate voltmeter. DCPD has to be privileged in the case of simple Paris' law determination for its simplicity and accuracy, at least for small to medium values of $a / W$. In multiaxial tests or plasticity studies, DIC methods have to be chosen. In addition, DIC allows further progress to be made in the study of inelastic effects by coupling the obtained full-fields and FEM calculations.

\section{ACKNOWLEDGEMENTS}

The authors wish to acknowledge the generous support of PSA Peugeot-Citroen, especially for the ductile cast iron provision.

\section{REFERENCES}

[1] A Wöhler. Versuche über die festigkeit der eisenbahnwagenachsen. Zeitschrift für Bauwesen, 10:160-161, 1860.

[2] $\mathrm{OH}$ Basquin. The exponential law of endurance tests. In Proc. Astm, volume 10, pages 625-630, 1910.

[3] SS Manson. Fatigue: a complex subject - some simple approximations. Experimental mechanics, 5(7):193-226, 1965.

[4] SS Manson. Behavior of materials under conditions of thermal stress. National Advisory Committee for Aeronautics, 1954.

[5] AA Griffith. The phenomena of rupture and flow in solids. Philosophical transactions of the royal society of london. Series A, containing papers of a mathematical or physical character, 221:163198, 1921.

[6] HM Westergaard. Bearing pressures and cracks. Journal of Applied Mechanics, 6:49-53, 1939.

[7] NI Muskhelishvili. Some Basic Problems of the Mathematical Theory of Elasticity. P. Noordhoff Ltd., Groningen, Holland, 1953.

[8] GR Irwin. Analysis of stresses and strains near the end of a crack traversing a plate. Journal of Applied Mechanics, 24(361-364):16, 1957.

[9] ML Williams. The bending stress distribution at the base of a stationary crack. Journal of Applied Mechanics, 28(1):78-82, 1961.

[10] PC Paris and F Erdogan. A critical analysis of crack propagation laws. Journal of basic engineering, 85(4):528-533, 1963.

[11] GL Greno, JL Otegui and RE Boeri. Mechanisms of fatigue crack growth in austempered ductile iron. International Journal of Fatigue, 21(1):35-43, 1999. 
[12] L Doremus, Y Nadot, G Henaff, C Mary and S Pierret. Calibration of the potential drop method for monitoring small crack growth from surface anomalies-crack front marking technique and finite element simulations. International Journal of Fatigue, 70:178-185, 2015.

[13] I Černỳ. The use of dcpd method for measurement of growth of cracks in large components at normal and elevated temperatures. Engineering Fracture Mechanics, 71(4):837-848, 2004.

[14] Y Murakami and LM Keer. Stress intensity factors handbook, vol. 3. Journal of Applied Mechanics, 60:1063, 1993.

[15] H Tada, PC Paris and GR Irwin. The analysis of cracks handbook. New York: ASME Press, 2:1, 2000 .

[16] JR Rice. A path independent integral and the approximate analysis of strain concentration by notches and cracks. Journal of applied mechanics, 35(2):379-386, 1968.

[17] J Réthoré, S Roux and F Hild. Noise-robust stress intensity factor determination from kinematic field measurements. Engineering Fracture Mechanics, 75(13):3763-3781, 2008.

[18] A El Bartali, V Aubin and S Degallaix. Fatigue damage analysis in a duplex stainless steel by digital image correlation technique. Fatigue $\mathcal{E}$ Fracture of Engineering Materials $\mathcal{E}$ Structures, 31(2):137-151, 2008.

[19] N Limodin, J Réthoré, JY Buffiere, F Hild, S Roux, W Ludwig, J Rannou and A Gravouil. Influence of closure on the $3 \mathrm{~d}$ propagation of fatigue cracks in a nodular cast iron investigated by x-ray tomography and 3d volume correlation. Acta Materialia, 58(8):2957-2967, 2010.

[20] J Abanto-Bueno and J Lambros. Investigation of crack growth in functionally graded materials using digital image correlation. Engineering Fracture Mechanics, 69(14):1695-1711, 2002.

[21] R Hamam, F Hild and S Roux. Stress intensity factor gauging by digital image correlation: Application in cyclic fatigue. Strain, 43(3):181-192, 2007.

[22] E Durif, J Rethore, A Combescure, M Fregonese and P Chaudet. Controlling stress intensity factors during a fatigue crack propagation using digital image correlation and a load shedding procedure. Experimental mechanics, 52(8):1021-1031, 2012.

[23] N Limodin, J Réthoré, JY Buffière, A Gravouil, F Hild and S Roux. Crack closure and stress intensity factor measurements in nodular graphite cast iron using three-dimensional correlation of laboratory x-ray microtomography images. Acta materialia, 57(14):4090-4101, 2009.

[24] J Lachambre, J Réthoré, A Weck and JY Buffiere. Extraction of stress intensity factors for $3 \mathrm{~d}$ small fatigue cracks using digital volume correlation and x-ray tomography. International Journal of Fatigue, 71:3-10, 2015.

[25] C Roux-Langlois, A Gravouil, MC Baietto, J Réthoré, F Mathieu, F Hild and S Roux. DIC identification and X-FEM simulation of fatigue crack growth based on the williams series. International Journal of Solids and Structures, 53:38-47, 2015.

[26] F Mathieu, F Hild and S Roux. Identification of a crack propagation law by digital image correlation. International Journal of Fatigue, 36(1):146-154, 2012. 
[27] E Charkaluk, A Bignonnet, A Constantinescu and K Dang Van. Fatigue design of structures under thermomechanical loadings. Fatigue \& Fracture of Engineering Materials \& Structures, 25(12):1199-1206, 2002.

[28] F Szmytka, H Maitournam and L Rémy. An implicit integration procedure for an elastoviscoplastic model and its application to thermomechanical fatigue design of automotive parts. Computers \& Structures, 119:155-165, 2013.

[29] GA Hartman and DA Johnson. Dc electric-potential method applied to thermal/mechanical fatigue crack growth. Experimental mechanics, 27(1):106-112, 1987.

[30] RP Gangloff, DC Slavik, RS Piascik and RH Van Stone. Direct current electrical potential measurement of the growth of small cracks. In Small-Crack Test Methods. ASTM International, 1992.

[31] RO Ritchie and KJ Bathe. On the calibration of the electrical potential technique for monitoring crack growth using finite element methods. International Journal of Fracture, 15(1):47-55, 1979.

[32] HH Johnson. Calibrating the electric potential method for studying slow crack growth (calibration of electric potential technique to study slow or steady crack growth in high strength materials). Materials Research and Standards, 5:442-445, 1965.

[33] G Clark and JF Knott. Measurement of fatigue cracks in notched specimens by means of theoretical electrical potential calibrations. Journal of the Mechanics and Physics of Solids, 23(4):265-276, 1975.

[34] MA Sutton, WJ Wolters, WH Peters, WF Ranson and SR McNeill. Determination of displacements using an improved digital correlation method. Image and vision computing, 1(3):133139, 1983.

[35] R Seghir, JF Witz, and S Courdert. Yadics digital image correlation 2/3D software. http: //yadics.univ-lille1.fr/.

[36] G Besnard, F Hild, and S Roux. Finite-element displacement fields analysis from digital images: application to portevin-le châtelier bands. Experimental Mechanics, 46(6):789-803, 2006.

[37] S Avril, M Bonnet, AS Bretelle, M Grediac, F Hild, P Ienny, F Latourte, D Lemosse, S Pagano, E Pagnacco and others. Overview of identification methods of mechanical parameters based on full-field measurements. Experimental Mechanics, 48(4):381-402, 2008.

[38] H Leclerc, JN Périé, S Roux and F Hild. Integrated digital image correlation for the identification of mechanical properties. In International Conference on Computer Vision/Computer Graphics Collaboration Techniques and Applications, pages 161-171. Springer, 2009.

[39] S Roux, J Réthoré and F Hild. Digital image correlation and fracture: an advanced technique for estimating stress intensity factors of $2 \mathrm{~d}$ and $3 \mathrm{~d}$ cracks. Journal of Physics D: Applied Physics, 42(21):214004, 2009.

[40] J Réthoré. Automatic crack tip detection and stress intensity factors estimation of curved cracks from digital images. International Journal for Numerical Methods in Engineering, 103(7):516-534, 2015. 
[41] C Henninger, S Roux and F Hild. Enriched kinematic of cracked structures. International Journal of Solids and Structures, 47:3305-3316, 2010.

[42] A Velichko and F Mücklich. Quantitative 3D characterisation of graphite morphology in cast iron - correlation between processing, microstructure and properties.

[43] GH Aronson and RO Ritchie. Optimization of the electrical potential technique for crack growth monitoring in compact test pieces using finite element analysis. Journal of Testing and Evaluation, 7(4):208-215, 1979.

[44] B Stokes, N Gao and PAS Reed. Effects of graphite nodules on crack growth behaviour of austempered ductile iron. Materials Science and Engineering: A, 445:374-385, 2007.

[45] F Hild and S Roux. Digital image correlation: from displacement measurement to identification of elastic properties-a review. Strain, 42(2):69-80, 2006.

[46] Y Nadot. Influence des défauts de fonderie sur la résistance à la fatigue d'une fonte GS. PhD thesis, 1997.

[47] P Clement, J Angeli and A Pineau. Short crack behaviour in nodular cast iron. Fatigue $\mathcal{E}$ Fracture of Engineering Materials \& Structures, 7(4):251-265, 1984.

[48] M Dahlberg. Fatigue crack propagation in nodular graphite cast iron. International Journal of Cast Metals Research, 17(1):29-37, 2004.

[49] T Ogawa and H Kobayashi. Near-threshold fatigue crack growth and crack closure in a nodular cast iron. Fatigue \& Fracture of Engineering Materials \& Structures, 10(4):273-280, 1987.

[50] P Dierickx. Etude de la microstructure et des mécanismes d'endommagement de fontes gs ductiles. influence des traitements thermiques de ferritisation. 1996.

[51] N Otsu. A threshold selection method from gray-level histograms. Automatica, 11(285296):23-27, 1975. 